\title{
Strong Integer Additive Set-valued Graphs: A Creative Review
}

\author{
N. K. Sudev \\ Department of Mathematics \\ Vidya Academy of Science \& Technology \\ Thalakkottukara, Thrissur-680501, India.
}

\author{
K. A. Germina \\ PG \& Research Department of Mathematics \\ Mary Matha Arts \& Science College \\ Mananthavady Wayanad-670645, India.
}

\author{
K. P. Chithra \\ Naduvath Mana, Nandikkara \\ Thrissur-680301, India.
}

\begin{abstract}
For a non-empty ground set $X$, finite or infinite, the set-valuation or set-labeling of a given graph $G$ is an injective function $f$ : $V(G) \rightarrow \mathcal{P}(X)$ such that the induced edge-function $f^{*}:$ $E(G) \rightarrow \mathcal{P}(X)-\{\emptyset\}$ is defined by $f^{*}(u v)=f(u) * f(v)$ for every $u v \in E(G)$, where $\mathcal{P}(X)$ is the power set of the set $X$ and $*$ is a binary operation on sets. A set-indexer of a graph $G$ is an set-labeling $f: V(G)$ such that the edge-function $f^{*}$ is also injective. An integer additive set-labeling (IASL) of a graph $G$ is defined as an injective function $f: V(G) \rightarrow \mathcal{P}\left(\mathbb{N}_{0}\right)$ such that the induced edge-function $g_{f}: E(G) \rightarrow \mathcal{P}\left(\mathbb{N}_{0}\right)$ is defined by $g_{f}(u v)=f(u)+f(v)$, where $\mathbb{N}_{0}$ is the set of all non-negative integers, $\mathcal{P}\left(\mathbb{N}_{0}\right)$ is its power set and $f(u)+f(v)$ is the sumset of the set-labels of two adjacent vertices $u$ and $v$ in $G$. An IASL $f$ is said to be a strong IASL if $\left|f^{+}(u v)\right|=|f(u)||f(v)|$ for every pair of adjacent vertices $u, v$ in $G$. In this paper, the characteristics and properties of strong integer additive set-labeled graphs are critically and creatively reviewed.
\end{abstract}

\section{Keywords:}

Integer additive set-labelings, integer additive set-indexers, strong integer additive set-labeling, strongly uniform integer additive setlabeling.

\section{AMS Subject Classification: $05 \mathrm{C78}$}

\section{PRELIMINARIES}

\subsection{Introduction to Set-Valued Graphs}

For all terms and definitions, not defined specifically in this paper, the reader may refer to [21]. For more about graph classes, one may further refer to [9] and [15]. Unless mentioned otherwise, all graphs considered here are simple, finite and have no isolated vertices.

The researches on graph labeling problems commenced with the introduction of the concept of number valuations of graphs in [26]. Since then, the studies on graph labeling have contributed significantly to the researches in graph theory and associated fields. Graph labeling problems have numerous theoretical and practical applications. Many types of graph labelings are surveyed and listed in [15].

Motivated from various problems related to social interactions and social networks, in [1], the notion of set-valuation of graphs has been introduced as an analogue of the number valuations of graphs. For a non-empty ground set $X$, finite or infinite, the setvaluation or set-labeling of a given graph $G$ is an injective function $f: V(G) \rightarrow \mathcal{P}(X)$, where $\mathcal{P}(X)$ is the power set of the set $X$.

Also, the notion of a set-indexer of a graph $G$ is also defined in [1] as an injective set-valued function $f: V(G) \rightarrow \mathcal{P}(X)$ such that the function $f^{*}: E(G) \rightarrow \mathcal{P}(X)-\{\emptyset\}$ defined by $f^{*}(u v)=$ $f(u) * f(v)$ for every $u v \in E(G)$ is also injective, where $\mathcal{P}(X)$ is the set of all subsets of $X$ and $*$ is a binary operation on sets.

Taking the symmetric difference of two sets as the operation between two set-labels of the vertices of $G$, the following theorem was proved in [1].

THEOREM 1.1. [1] Every graph has a set-indexer.

\subsection{Integer Additive Set-Valued Graphs}

The sumset of two non-empty sets $A$ and $B$, denoted by $A+B$, is the set defined by $A+B=\{a+b: a \in A, b \in B\}$. If the set $C$ is the sumset of two sets $A$ and $B$, then $A$ and $B$ are said to be the summands of $C$. It is to be noted that any non-empty set $A$ can be expressed as $A=\{0\}+A$. Here, $A$ and $\{0\}$ are said to be trivial summands of $A$. In this paper, sumsets and summands mentioned are non-trivial sumsets and non-trivial summands.

A useful theorem on the cardinality of the sumset of two sets, in terms of the summands, are proved in [23] as follows.

THEOREM 1.2. If $A$ and $B$ are two non-empty sets of integers, then $|A|+|B|-1 \leq|A+B| \leq|A||B|$.

Using the concepts of sumsets of sets, the notion of integer additive set-labeling was introduced as follows.

Definition 1.3. 16 27 Let $\mathbb{N}_{0}$ denote the set of all nonnegative integers and $\mathcal{P}\left(\mathbb{N}_{0}\right)$ be its power set. An integer additive set-labeling (IASL, in short) of a graph $G$ is defined as an injective function $f: V(G) \rightarrow \mathcal{P}\left(\mathbb{N}_{0}\right)$ which induces a function $f^{+}$: $E(G) \rightarrow \mathcal{P}\left(\mathbb{N}_{0}\right)$ such that $f^{+}(u v)=f(u)+f(v), u v \in E(G)$.

A Graph which admits an IASL is called an integer additive setlabeled graph (IASL-graph).

The notion of integer additive set-indexers of graphs are introduced as follows. 
DEFINITION 1.4. [16] An integer additive set-labeling $f$ : $V(G) \rightarrow \mathcal{P}\left(\mathbb{N}_{0}\right)$ of a graph $G$ is said to be an integer additive set-indexer (IASI) if the induced function $f^{+}: E(G) \rightarrow \mathcal{P}\left(\mathbb{N}_{0}\right)$ defined by $f^{+}(u v)=f(u)+f(v)$ is also injective.

A Graph which admits an IASI is called an integer additive setindexed graph (IASI-graph).

An IASL (or IASI) is said to be $k$-uniform if $\left|f^{+}(e)\right|=k$ for all $e \in E(G)$. That is, a connected graph $G$ is said to have a $k$-uniform IASL (or IASI) if all of its edges have the same set-indexing number $k$. If $G$ is a graph which admits a $k$-uniform IASI and $V(G)$ is $l$-uniformly set-indexed, then $G$ is said to have a $(k, l)$-completely uniform IASI or simply a completely uniform IASI.

If either $f(u)$ or $f(v)$ is countably infinite, then clearly their sumset will also be countably infinite and hence the study of the cardinality of the sum set $f(u)+f(v)$ becomes trivial. Hence, this discussion is restricted to finite sets of non-negative integers. We denote the cardinality of a set $A$ by $|A|$. The cardinality of the set-label of an element (vertex or edge) of a graph $G$ is called the set-indexing number of that element. If the set-labels of all vertices of $G$ have the same cardinality, then the vertex set $V(G)$ is said to be uniformly set-indexed.

Figure 1 illustrates a graph together with the integer additive setlabeling defined on it.

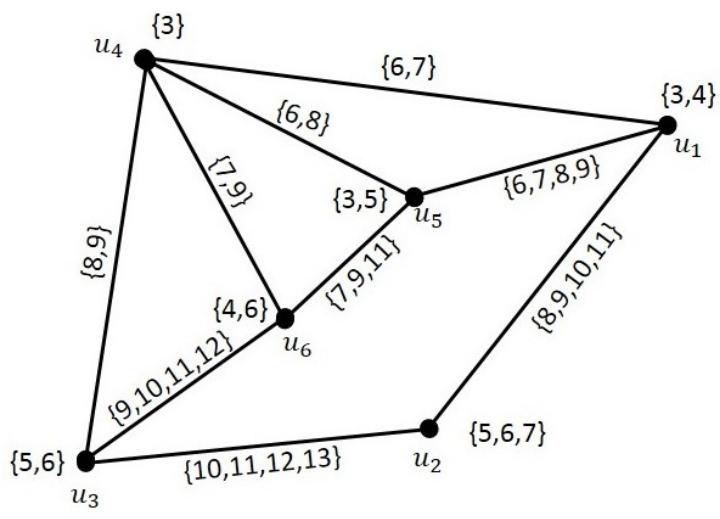

Fig. 1. A graph with an IASL defined on it

Certain studies about integer additive set-indexed graphs have been done in [4], [17], [28] and [27].

Analogous to Theorem 1.1 the following theorem has been proved in [27].

THEOREM 1.5. [27] Every graph has an integer additive setlabeling (or an integer additive set-indexer).

\section{STRONG INTEGER ADDITIVE SET-LABELED GRAPHS}

The cardinality of the sum set of two non-empty finite sets $A$ and $B$ is always less than or equal to the product $|A||B|$. The study regarding the characteristics of graphs in which the set-indexing number of every edge is the product of the set-indexing numbers of its end vertices arises much interest. Hence, the following terms are defined.
DEFINITION 2.1. [28] If a graph $G$ has a set-labeling $f$ such that $\left|f^{+}(u v)\right|=|f(u)+f(v)|=|f(u)||f(v)|$ for any pair of adjacent vertices $u$ and $v$ of $G$, then $f$ is said to be a strong integer additive set-labeling (strong IASL) of $G$. A graph which admits a strong IASL is called a strong IASL-graph.

DEFINITION 2.2. [28] An IASI $f: V(G) \rightarrow \mathcal{P}\left(\mathbb{N}_{0}\right)$ of a given graph $G$, such that $\left|f^{+}(u v)\right|=|f(u)+f(v)|=|f(u)||f(v)|$ for any pair of adjacent vertices $u$ and $v$ of $G$, is said to be a strong $I A S I$ of $G$. A graph which admits a strong IASI is called a strong IASI-graph.

Figure 2 depicts a graph with a strong integer additive set-indexer defined on it.

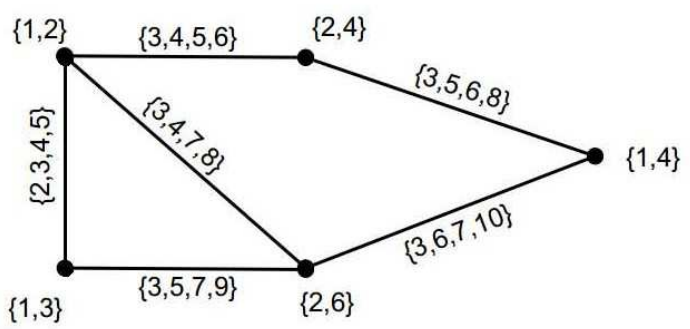

Fig. 2. An example to a Strong IASI-graph.

Let $A$ be a non-empty finite set of non-negative integers. The difference set of $A$, denoted by $D_{A}$, is the set defined by $D_{A}=\{|a-b|$ : $a, b \in A\}$. A necessary and sufficient condition for a graph $G$ to admit a strong IASI, in terms of the difference sets of the set-labels of the vertices of a given graph, has been established in [28] as follows.

THEOREM 2.3. [28] A graph $G$ admits a strong IASI if and only if the difference sets of the set-labels of any two adjacent vertices in $G$ are disjoint.

The notation $A<B$ is generally used to indicate that the sets $A$ and $B$ are mutually disjoint sets. By the sequence $A_{1}<A_{2}<$ $A_{3}<\ldots<A_{n}$, it is meant that the given sets are pairwise disjoint. The relation $<$ is called the difference relation of $G$ and the length of a sequence of difference sets is the number of difference sets in that sequence. Then, a necessary and sufficient condition for a given graph to admit a strong IASL (or strong IASI) is given below.

THEOREM 2.4. [28] Let $G$ be a connected graph on $n$ vertices. Then, $G$ admits a strong IASI if and only if there exists a finite sequence of difference sets $D_{1}<D_{2}<D_{3}<\ldots<D_{m} ; m \leq n$, where $D_{i}$ is the difference set of the set-label a vertex $v_{i}$ of $G$.

By Theorem 2.3. if $G$ admits a strong IASI $f$, then $D_{f(u)}<D_{f(v)}$, for any two adjacent vertices $u$ and $v$ of $G$. The relation $<$ forms one or more sequence of difference sets for $G$. Then, the following notion has been introduced.

Definition 2.5. [29] The nourishing number of a set-labeled graph is the minimum length of the maximal sequence of difference sets of the set-labels of the vertices in $G$. The nourishing number of a graph $G$ is denoted by $\varkappa(G)$.

In other words, the nourishing number of a graph $G$ is the minimum order of a maximal connected subgraph of $G$, the difference sets of the set-labels of whose vertices are necessarily pairwise disjoint. 
In view of the above notions, a necessary and sufficient condition for a complete graph $G$ is given below.

THEOREM 2.6. [28] A complete graph $K_{n}$ admits a strong IASL (or IASI) if and only if the difference sets of the set-labels of all vertices of $G$ are pairwise disjoint.

The following theorem establishes the hereditary nature of the existence of a strong IASL (or a strong IASI).

THEOREM 2.7. Any subgraph of a strong IASL-graph also admits a (induced) strong IASI.

That is, for a vertex $v_{i}$ of a complete graph $K_{n}$, if $D_{i}$ is the difference set of $f\left(v_{i}\right)$, then clearly $D_{1}<D_{2}<D_{3}<\ldots<D_{n}$. Therefore, the following result is immediate.

Figure 3 establishes the existence of an induced strong IASI for the subgraph $H$ of a given strong IASI-graph $G$.
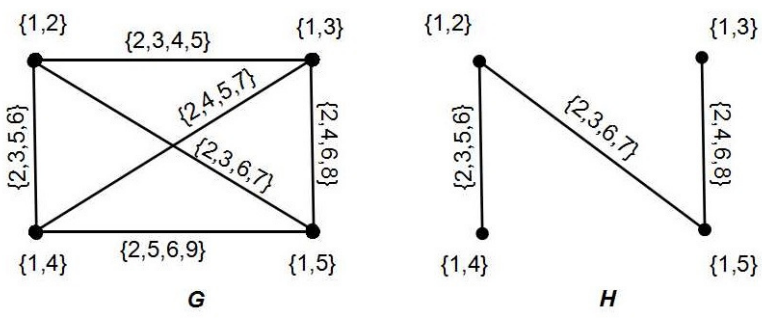

Fig. 3. An illustration to the hereditary nature of the existence of a strong IASI.

PROPOSITION 2.8. [29] The nourishing number of a complete graph $K_{n}$ is $n$.

The following results are on the nourishing number of different graphs.

PROPOSITION 2.9. [29] The nourishing number of a bipartite graph is 2 .

Proposition 2.10. [29] The nourishing number of a triangle-free graph is 2 .

Recall that a clique of graph $G$ is a complete subgraph of $G$ and the clique number, denoted by $\omega(G)$, of $G$ is the number of vertices in a maximal clique of $G$. Then, due to the above results, the theorem given below has been established.

THEOREM 2.11. [29] The nourishing number of a graph $G$ is the clique number $\omega(G)$ of $G$.

Invoking Theorem 2.11 the following results on various operations of graphs have been established. The following result is about the admissibility of a strong IASI for the union of two strong IASIgraphs.

THEOREM 2.12. [29] The union $G_{1} \cup G_{2}$ of two graphs $G_{1}$ and $G_{2}$, admits a strong IASI if and only if both $G_{1}$ and $G_{2}$ admit strong IASIs.

Invoking Theorem 2.11 one can have

THEOREM 2.13. [29] Let $G_{1}$ and $G_{2}$ be two strong IASIgraphs. Then, $\varkappa\left(G_{1} \cup G_{2}\right) \geq \max \left\{\varkappa\left(G_{1}\right), \varkappa\left(G_{2}\right)\right\}$.
The following theorem on the existence of a strong IASI by the join of two strong IASI-graphs has been established in [28] as follows.

THEOREM 2.14. [29] The join of two strong IASI-graphs admits a strong IASI if and only if the difference set of the set-label of every vertex of one of them is disjoint from the difference sets of the set-labels of all vertices of the other.

The nourishing number of the join two strong IASI-graphs is given in terms of the nourishing numbers of these graphs as

THEOREM 2.15. [29] Let $G_{1}$ and $G_{2}$ be two strong IASIgraphs. Then, $\varkappa\left(G_{1}+G_{2}\right)=\varkappa\left(G_{1}\right)+\varkappa\left(G_{2}\right)$.

We are now going to review the conditions for the existence of a strong IASI for the complement of a strong IASI-graph. Since $G$ and its complement $\bar{G}$ has the same vertex set, the vertices of $G$ and $\bar{G}$ must have the same set-labels. It can be noted that a strong IASI defined on a graph $G$ need not be a strong IASI for $\bar{G}$. Hence, a necessary and sufficient condition for the existence of a strong IASI for the complement of a strong IASI-graph has been proved as follows.

THEOREM 2.16. [29] The complement of a strong IASI-graph $G$ admits a strong IASI if and only if the difference sets of the setlabels of all vertices of $G$ are pairwise disjoint.

Then, the following is an immediate consequence of the above theorem.

PROPOSITION 2.17. If the complement $\bar{G}$ of a strong IASIgraph admits a strong IASI, then $\varkappa(G)=\varkappa(\bar{G})=|V(G)|$.

COROLLARY 2.18. If $G$ is a self-complementary graph on $n$ vertices, which admits a strong IASI, then $\varkappa(G)=n$.

The first graph in Figure 4 is an example to a strong IASI-graph and second graph in the figure is its complement which admits the induced strong IASI.
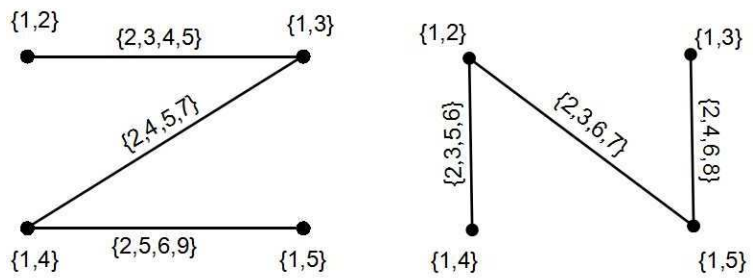

Fig. 4. An Example to a strong IASI-graph and its complement which admits an induced strong IASI.

The existence of strong IASI for certain products of graphs have also been established in [29]. The admissibility of a strong IASI by the Cartesian product of two strong IASI-graphs has been established in [29] as

THEOREM 2.19. [29] Let $G_{1}$ and $G_{2}$ be two strong IASIgraphs. Then, the product $G_{1} \square G_{2}$ admits a strong IASI if and only if the set-labels of corresponding vertices different copies of $G_{1}$ which are adjacent in $G_{1} \square G_{2}$ are disjoint.

Proposition 2.20. Let $G_{1}$ and $G_{2}$ be two graphs which admit strong IASIs. Then, $\varkappa\left(G_{1} \square G_{2}\right)=\max \left\{\varkappa\left(G_{1}\right), \varkappa\left(G_{2}\right)\right\}$.

In [29], the admissibility of a strong IASI by the corona of two strong IASI-graphs has been established as follows. 
THEOREM 2.21. Let $G_{1}$ and $G_{2}$ be two strong IASI-graphs. Then, their corona $G_{1} \odot G_{2}$ admits a strong IASI if and only if the difference set of the set-label of every vertex of $G_{1}$ is disjoint from the difference sets of the set-labels of all vertices of the corresponding copy of $G_{2}$.

The nourishing number of the corona of two graphs is given in the following result, in terms of the nourishing number of the individual graphs, as follows.

PROPOSITION 2.22. If $G_{1}$ and $G_{2}$ are two strong IASI-graphs, then

$$
\varkappa\left(G_{1} \odot G_{2}\right)= \begin{cases}\varkappa\left(G_{1}\right) & \text { if } \varkappa\left(G_{1}\right)>\varkappa\left(G_{2}\right) \\ \varkappa\left(G_{2}\right)+1 & \text { if } \varkappa\left(G_{2}\right)>\varkappa\left(G_{1}\right)\end{cases}
$$

\section{ASSOCIATED GRAPHS OF STRONG IASI GRAPHS}

In this section, it is intended to review the results on the admissibility of induced strong IASIs by certain graphs that are associated to given strong IASI-graphs .

The line graph of an undirected graph $G$ is another graph $L(G)$ that represents the adjacencies between edges of $G$. That is, every edge of $G$ corresponds to a vertex in $L(G)$ and the adjacency of edges in $G$ defines the adjacency of corresponding vertices in $L(G)$. Hence, one may find the following result.

THEOREM 3.1. 27] The line graph of a strong IASI-graph does not admit an induced strong IASI.

The proof of the above theorem follows from the facts that $D_{f(u)} \cup$ $D_{f(v)} \subseteq D_{f+(u v)}$ for any two adjacent vertices $u$ and $v$ in $G$ and for any two adjacent edges $e_{1}$ and $e_{2}$ in $G, D_{f^{+}\left(e_{1}\right)} \cap D_{f+\left(e_{2}\right)} \neq \emptyset$.

The total graph $T(G)$ of a graph $G$ has a vertex corresponding to each element (edge or vertex) of $G$ such that the adjacency between the vertices in $T(G)$ is determined by the adjacency or incidence between the corresponding elements of $G_{i}$. Then, the following result have been observed.

THEOREM 3.2. 27| The total graph of a strong IASI-graph does not admit an induced strong IASI.

The line graph of $G$ is a subgraph of the total graph $G$ and by Theorem 3.1 $L(G)$ does not admit a strong IASI. Hence, by Theorem $2.7 T(G)$ does not admit a strong IASI.

A graph $H$ is said to be homeomorphic to a given graph $G$ if $H$ is obtained by replacing some paths of length 2 in $G$ which are not in any triangle of $G$, by some edges (which are originally not in $G$ ). This operation is called an elementary topological reduction. The following is a necessary and sufficient condition for a graph homeomorphic to a given strong IASI-graph $G$ to admit a strong IASI.

THEOREM 3.3. [28] Any graph H, obtained by applying a $r$ elementary topological reductions on a strong IASI-graph $G$, admits a strong IASI if and only if there exist $r$ distinct paths of length 2 in $G$ which are not in any triangle of $G$, the difference sets of the set-labels of whose vertices are pairwise disjoint.

Figure 5 depicts the admissibility of an induced strong IASI for the homeomorphic images of $G=K_{4}$.

A graph $H$ is a minor of a graph $G$ if it can be obtained from a subgraph of $G$ by contracting some of its edges. Here, contracting an edge $e$ means deleting $e$ and then identifying its end vertices.

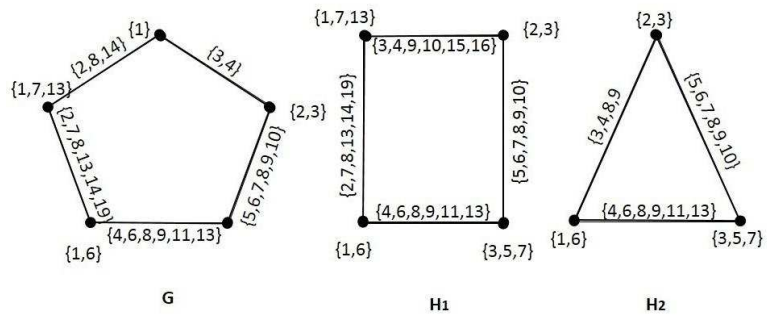

Fig. 5. Admissibility of an induced strong IASI for the graphs that are homeomorphic to a given strong IASI-graph.

We now propose the the following results on the admissibility of strong IASI by the minors of given strong IASI-graphs.

THEOREM 3.4. A minor $H$ of a strong IASI-graph admits an induced strong IASI if and only if there exists at least one edge in $G$, the difference set of the set-label of which is disjoint from the difference sets of the set-labels of its neighbouring vertices.

Proof. Let $G$ be a graph that admits a strong IASI $f$ and let $e=v_{i} v_{j}$ be an edge of $G$. Let $H$ be a graph obtained by contracting the edge $e$. If $w$ is the new vertex in $H$ obtained by identifying the end vertices of $e$ in $G$. Then, the set-label of $w$ in $H$ is the same as that of $e$ in $G$. Note that all vertices of $G$ other than $v_{i}$ and $v_{j}$ are also the vertices of $H$. Let $v_{r}$ and $v_{s}$ be the vertices adjacent to $v_{i}$ and $v_{j}$ respectively, in $G$. Then, $v_{r}$ and $v_{s}$ be the adjacent vertices of $w$ in $H$.

Now, assume that $H$ admits a strong IASI, say $f^{\prime}$, induced by $f$. Then, for all vertices $v \neq v_{i}, v_{j}$, one may have $f^{\prime}(v)=f(v)$ and $f^{\prime}(w)=f^{+}(u v)$. Therefore, $D_{f^{\prime}(w)} \cap D_{f\left(v_{r}\right)}=\emptyset$ and $D_{f^{\prime}(w)} \cap$ $D_{f\left(v_{s}\right)}=\emptyset$ and hence $D_{f+\left(v_{i} v_{j}\right)} \cap D_{f\left(v_{r}\right)}=\emptyset$ and $D_{f+\left(v_{i} v_{j}\right)} \cap$ $D_{f\left(v_{s}\right)}=\emptyset$.

To prove the converse, assume that $D_{f+\left(v_{i} v_{j}\right)} \cap D_{f\left(v_{r}\right)}=\emptyset$ and $D_{f+\left(v_{i} v_{j}\right)} \cap D_{f\left(v_{s}\right)}=\emptyset$. Now, let $f^{\prime}$ be the IASI on $H$ induced by $f$. Then, $f^{\prime}(w)=f^{+}\left(v_{i} v_{j}\right)$ and hence $D_{f^{\prime}(w)} \cap D_{f\left(v_{r}\right)}=\emptyset$ and $D_{f^{\prime}(w)} \cap D_{f\left(v_{s}\right)}=\emptyset$. Since all other edges $u v$ of $H$ are the edges of $G$ and $f$ is a strong IASI of $G$, it can be noticed that $D_{f^{\prime}(u)} \cap D_{f^{\prime}(u)}=D_{f(u)} \cap D_{f(u)}=\emptyset$. Therefore, $f^{\prime}$ is a strong IASI of $H$ induced by the strong IASI $f$ of $G$.

A graph $H$ is a subdivision of a graph $G$ if $H$ can be obtained from $G$ by replacing some of its edges with some paths of length 2 such that the inner vertices of these paths have degree 2 in $H$. The following theorem verifies whether a subdivision of $G$ admits a strong IASL.

THEOREM 3.5. A subdivision $S(G)$ of a strong IASI-graph $G$ does not admit an induced strong IASI.

Proof. Let the given graph $G$ admits a strong IASI $f$. Since $f$ is a strong IASI of $G$, for any two adjacent vertices $u$ and $v$ in $G D_{f(u)} \cup D_{f(v)} \subseteq D_{f+(u v)}$. Let $P_{2}: v_{i} v_{j} v_{k}$ be a path in $G$. Now, let $H$ be a graph obtained by replacing the edge $u v$ by a path $u w v$. Here, under an IASI, induced by $f$, the new element $w$ takes the same set-label of the removed edge $u v$. Therefore, $D_{f(u)} \cap$ $D_{f(w)}=D_{f(u)} \neq \emptyset$. Similarly, $D_{f(w)} \cap D_{f(v)}=D_{f(v)} \neq \emptyset$. Hence, $f$ does not induce a strong IASI on $H$. 


\section{NOURISHING NUMBER OF GRAPH POWERS}

In this section, the discussion was on the nourishing number of cetain graph classes and their powers. For a positive integer $r$, the $r$-th power of a simple graph $G$ is the graph $G^{r}$ whose vertex set is $V$, two distinct vertices being adjacent in $G^{r}$ if and only if their distance in $G$ is at most $r$. The graph $G^{2}$ is referred to as the square of $G$, the graph $G^{3}$ as the cube of $\mathrm{G}$. To proceed further, one may need the following theorem on graph powers.

THEOREM 4.1. [31] If $d$ is the diameter of a graph $G$, then $G^{d}$ is a complete graph.

Let us begin with the following result on the nourishing number of the finite powers of complete bipartite graphs.

THEOREM 4.2. [30] The nourishing number of the $r$-th power of a complete bipartite graph is

$$
\varkappa\left(K_{m, n}^{r}\right)= \begin{cases}2 & \text { if } r=1 \\ m+n & \text { if } r \geq 2\end{cases}
$$

The proof is immediate from the fact that the diameter of a complete bipartite graph is 2 .

The nourishing number of a path has been estimated in [30] as follows.

THEOREM 4.3. [30] The nourishing number of the $r$-th power of a path $P_{m}$ is

$$
\varkappa\left(P_{m}\right)= \begin{cases}r+1 & \text { if } r<m \\ m+1 & \text { if } r \geq m .\end{cases}
$$

The following result is on the nourishing number of the $r$-th power of a cycle.

THEOREM 4.4. [30] The nourishing number of the $r$-th power of a cycle $C_{n}$ is

$$
\varkappa\left(C_{n}\right)= \begin{cases}r+1 & \text { if } r<\left\lfloor\frac{n}{2}\right\rfloor \\ n & \text { if } r \geq\left\lfloor\frac{n}{2}\right\rfloor .\end{cases}
$$

A wheel graph, denoted by $W_{n+1}$, is defined as $W_{n+1}=C_{n}+K_{1}$. The nourishing number of the powers of wheel graphs is discussed in the following proposition.

THEOREM 4.5. [30] The nourishing number of the $r$-th power of a wheel graph is

$$
\varkappa\left(W_{n+1}^{r}\right)= \begin{cases}3 & \text { if } r=1 \\ n+1 & \text { if } r \geq 2 .\end{cases}
$$

A helm graph, denoted by $H_{n}$, is a graph obtained by adjoining a pendant edge to each of the vertices of the outer cycle of a wheel graph $W_{n+1}$. A helm graph has $2 n+1$ vertices and $3 n$ edges. The following theorem is on the nourishing number of a helm graph and its powers.

THEOREM 4.6. [30] The nourishing number of the $r$-th power of a helm graph $H_{n}$ is given by

$$
\varkappa H_{n}^{r}= \begin{cases}3 & \text { if } r=1 \\ n+1 & \text { if } r=2 \\ n+4 & \text { if } r=3 \\ 2 n+1 & \text { if } r \geq 4\end{cases}
$$

Another graph that is being considered in this context is a friendship graph $F_{n}$, which is the graph obtained by joining $n$ copies of the cycle $C_{3}$ with a common vertex. It has $2 n+1$ vertices and $3 n$ edges. The following proposition is about the nourishing number of a friendship graph $F_{n}$.

THEOREM 4.7. [30] The nourishing number of the $r$-th power of a friendship graph $F_{n}$ is

$$
\varkappa\left(F_{n}^{r}\right)= \begin{cases}3 & \text { if } r=1 \\ 2 n+1 & \text { if } r \geq 2\end{cases}
$$

Another similar graph structure is a fan graph $F_{m, n}$, which is defined as $\bar{K}_{m}+P_{n}$. The nourishing number of a fan graph $F_{m, n}$ and its powers are estimated as follows.

THEOREM 4.8. [30] The nourishing number of the $r$-th power of a fan graph $F_{m, n}$ is

$$
\varkappa\left(F_{m, n}^{r}\right)= \begin{cases}3 & \text { if } r=1 \\ m+n & \text { if } r \geq 2\end{cases}
$$

A split graph, denoted by $G\left(K_{r}, S\right)$, is a graph in which the vertices can be partitioned into a clique $K_{r}$ and an independent set $S$. A split graph is said to be a complete split graph if every vertex of the independent set $S$ is adjacent to every vertex of the the clique $K_{r}$ and is denoted by $K_{S}(r, s)$, where $r$ and $s$ are the orders of $K_{r}$ and $S$ respectively. The nourishing number of a split graph is estimated in the following theorem.

THEOREM 4.9. [30] The nourishing number of the $r$-th power of a split graph $G=G\left(K_{r}, S\right)^{r}$ is given by

$\varkappa\left(G^{r}\right)= \begin{cases}r & \text { if no vertex of } S \text { is adjacent to all vertices of } K_{r} \\ r+1 & \text { if some vertices of } S \text { are adjacent to all vertices of } K_{r} \\ r+l & \text { if } r=2 \\ r+s & \text { if } r \geq 3 ;\end{cases}$

In view of Theorem 4.9, the following result on complete split graphs have been established.

COROLlaRY 4.10. The nourishing number of the $k$-th power of a complete split graph $G=K_{S}(r, s)$ is given by

$$
\varkappa\left(G^{r}\right)= \begin{cases}r+1 & \text { if } k=1 \\ r+s & \text { if } k \geq 2 ;\end{cases}
$$

An $n$-sun or a trampoline, denoted by $S_{n}$, is a chordal graph on $2 n$ vertices, where $n \geq 3$, whose vertex set can be partitioned into two sets $U=\left\{u_{1}, u_{2}, u_{3}, \ldots, u_{n}\right\}$ and $W=\left\{w_{1}, w_{2}, w_{3}, \ldots, w_{n}\right\}$ such that $W$ is an independent set of $G$ and $w_{j}$ is adjacent to $u_{i}$ if and only if $j=i$ or $j=i+1(\bmod n)$. A complete sun is a sun $G$ where the induced subgraph $\langle U\rangle$ is complete. The sun graphs with $\langle U\rangle$ is a cycle is one of the most interesting structures among the sun graphs. The following theorem determines the nourishing number of a sun graph $G$ whose non-independent set of vertices induces a cycle in the graph $G$.

THEOREM 4.11. [30] If $G$ is an n-sun graph with $\langle U\rangle=C_{n}$, then the nourishing number of $G^{r}$ is given by

$$
\varkappa\left(G^{r}\right)= \begin{cases}2 r+1 & \text { if } r<\left\lfloor\frac{n}{2}\right\rfloor \\ 2(n-1) & \text { if } r=\left\lfloor\frac{n}{2}\right\rfloor \text { and if } n \text { is odd } \\ 2 n-1 & \text { if } r=\left\lfloor\frac{n}{2}\right\rfloor \text { and if } n \text { is even } \\ 2 n & \text { if } r \geq\left\lfloor\frac{n}{2}\right\rfloor+1 ;\end{cases}
$$


THEOREM 4.12. [30] The nourishing number of a complete nsun graph $G$ is given by

$$
\varkappa\left(G^{r}\right)= \begin{cases}n & \text { if } r=1 \text { and if }\langle U\rangle \text { is triangle-free } \\ n+1 & \text { if } r=2 \\ 2 n & \text { if } r \geq 3 ;\end{cases}
$$

Another important graph is an $n$-sunlet graph, which is the graph on $2 n$ vertices obtained by attaching one pendant edge to each vertex of a cycle $C_{n}$. The following result discusses about the nourishing number of an $n$-sunlet graph.

THEOREM 4.13. [30] The nourishing number of the $r$-th power of an $n$-sunlet graph $G$ is

$$
\varkappa\left(G^{r}\right)= \begin{cases}2 r & \text { if } r<\left\lfloor\frac{n}{2}\right\rfloor+1 \\ 2(n-1) & \text { if } r=\left\lfloor\frac{n}{2}\right\rfloor+1 \text { and } n \text { is odd } \\ 2 n-1 & \text { if } r=\left\lfloor\frac{n}{2}\right\rfloor+1 \text { and } n \text { is even } \\ 2 n & \text { if } r \geq\left\lfloor\frac{n}{2}\right\rfloor+2 .\end{cases}
$$

\section{STRONGLY UNIFORM IASI GRAPHS}

Definition 5.1. [28] A graph $G$ is said to have a strongly uniform IASI if there exists an IASI $f$ defined on $G$ such that $\left|f^{+}(u v)\right|=k=|f(u)||f(v)|$ for all $u, v \in V(G)$, where $k$ is a positive integer.

Figure 6 illustrates some graphs which admit strongly uniform integer additive set-indexers.

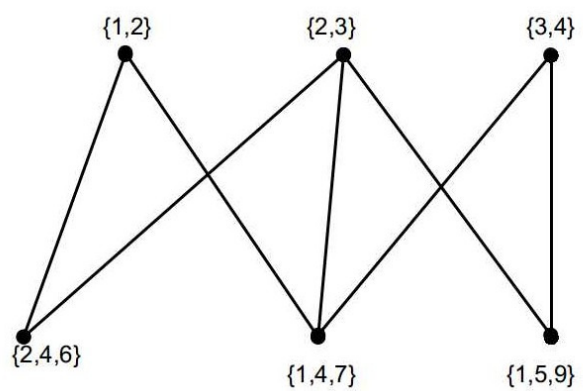

Fig. 6. Examples to Strongly Uniform IASI-Graphs

In view of the above definition, it can be noticed that if a graph $G$ admits a strongly $k$-uniform IASI, then every edge of $G$ has the setindexing number which is the product of the set-indexing numbers of its end vertices. Hence, if $G$ is a strongly $k$-uniform IASI-graph, then each vertex of $G$ has some set-indexing number $d_{i}$, which is a divisor of $k$. Hence, $V(G)$ can be partitioned into at most $n$ sets, say $\left(X_{1}, X_{2}, \ldots, X_{n}\right)$ such that each $X_{i}$ consists of the vertices of $G$ having the set-indexing number $d_{i}$, where $n$ is the number of divisors of the integer $k$.

The admissibility of strongly uniform IASIs by bipartite graphs had been established in the following theorem.

THEOREM 5.2. [28] For any positive integer $k$, all bipartite graphs admit a strongly $k$-uniform IASI.

The following theorem has established a condition required for a complete graph to admit a strongly $k$-uniform IASI.
THEOREM 5.3. [28] A strongly $k$ - uniform IASI of a complete graph $K_{n}$ is a $(k, l)$-completely uniform IASI, where $l=\sqrt{k}$.

Now, recall the following fact from the number theory. The number of divisors of a non-square integer is even and the number of divisors of a perfect square is odd. Then, the following result can be observed.

THEOREM 5.4. [28] For a positive integer $k$, let $G$ be a graph which admits a strongly $k$-uniform IASI. Also, let $n$ be the number of divisors of $k$. Then, if $k$ is not a perfect square, then $G$ has at most $\frac{n}{2}$ bipartite components and if $k$ is a perfect square, then $G$ has at most of $\frac{n+1}{2}$ components in which at most $\frac{n-1}{2}$ components are bipartite components.

Invoking Theorem 5.4 it can be noted that if the vertex set of a graph $G$ admitting a strongly $k$-uniform IASI, can be partitioned into more than two sets, then $G$ is a disconnected graph. Hence, a more generalised result can be obtained as follows.

THEOREM 5.5. [28] Let $k$ be a non-square integer. Then a graph $G$ admits a strongly k-uniform IASI if and only if $G$ is bipartite or a union of disjoint bipartite components.

The following results are immediate consequences of the above results.

THEOREM 5.6. [28] If a graph $G$, which admits a strongly $k$ uniform IASI, then it contains at most one component which is a clique.

THEOREM 5.7. [28] Let the graph $G$ has a strongly $k$-uniform IASI. Hence, if $G$ has a component which is a clique, then $k$ is a perfect square.

Hence, One can easily follow the theorem given below.

THEOREM 5.8. [28] A connected non-bipartite graph $G$ admits a strongly $k$-uniform IASI if and only if $k$ is a perfect square and this IASI is a $(k, l)$-completely uniform, where $l=\sqrt{k}$.

From all of our discussions about the strongly uniform IASIgraphs, one can establish a generalised result as follows.

THEOREM 5.9. [28] A connected graph $G$ admits a strongly $k$-uniform IASI if and only if $G$ is bipartite or this IASI is a $(k, l)$ completely uniform IASI of $G$, where $k=l^{2}$.

\section{CONCLUSION SCOPE FOR FURTHER STUDIES}

In this paper, some characteristics of the graphs which admit strong IASIs and strongly $k$-uniform IASIs have been given. More studies are possible in this area when the ground set $X$ is finite instead of the set $\mathbb{N}_{0}$.

The problems of establishing the necessary and sufficient conditions for various graphs and graph classes to admit these types of uniform IASIs have still been unsettled. The existence of strong IASI, both uniform and non-uniform, for certain other graph products is also woth-studying.

Certain IASIs which assign set-labels having specific properties, to the elements of given graphs also seem to be promising for further investigations. All these facts highlight the wide scope for intensive studies in this area. 


\section{Acknowledgement}

The concept of integer additive set-indexers of graphs was first introduced by Professor Belamannu Devadas Acharya during a personal communication with the second author of this paper. The authors dedicate this paper to the bright memory this great mathematician.

\section{REFERENCES}

[1] B. D. Acharya, Set-Valuations and Their Applications, MRI Lecture notes in Applied Mathematics, No.2, The Mehta Research Institute of Mathematics and Mathematical Physics, Allahabad, 1983.

[2] B. D. Acharya, Set-Indexers of a Graph and Set-Graceful Graphs, Bull. Allahabad Mathematical Society, 16(2001), 123.

[3] B. D. Acharya and K. A. Germina, Strongly Indexable Graphs: Some New Perspectives, Advanced Modelling and Optimisation, 15(1)(2013), 3-22.

[4] T. M. K. Anandavally, A Characterisation of 2-Uniform IASI Graphs, International Journal of Contemporary Mathematical Sciences, 8(10)(2013), 459-462.

[5] T. M. Apostol, Introduction to Analytic Number Theory, Springer-Verlag, New York, 1989.

[6] M. Behzad, The connectivity of Total Graphs, Bulletin of Australian Mathematical Society, 1(1969), 175-181.

[7] J. A. Bondy and U. S. R. Murty, Graph Theory with Applications, Macmillan Press, London, 1976.

[8] J. A. Bondy and U. S. R. Murty, Graph Theory, Springer, 2008.

[9] A. Brandstädt, V. B. Le and J. P. Spinrad, Graph Classes:A Survey, SIAM, Philadelphia, 1999.

[10] A. Brandädt, P. L. Hammer, V. B. Le and V. L. Lozin, Bisplit graphs, Discrete Mathematics, 299(1-3)(2005), 11-32.

[11] D. M. Burton, Elementary Number Theory, Tata McGrawHill Inc., New Delhi, 2007.

[12] M. Capobianco and J. Molluzzo, Examples and Counterexamples in Graph Theory, North-Holland, New York, 1978.

[13] N. Deo, Graph theory With Application to Engineering and Computer Science, Prentice Hall, India, 1974.

[14] R. Frucht and F. Harary, On the Corona of Two Graphs, Aequationes Mathemaematica, 4(3)(1970), 322-325.

[15] J. A. Gallian, A Dynamic Survey of Graph Labelling, The Electronic Journal of Combinatorics (DS \#6), 2011.

[16] K. A. Germina and T. M. K. Anandavally, Integer Additive Set-Indexers of a Graph: Sum Square Graphs, Journal of Combinatorics, Information and System Sciences, 37(24)(2012), 345-358.

[17] K. A. Germina, N. K. Sudev, On Weakly Uniform Integer Additive Set-Indexers of Graphs, International Mathematical Forum, 8(37-40)(2013), 1827-1834.

[18] J. T. Gross and J. Yellen, Graph Theory and its Applications, CRC Press, 2006.

[19] G Hahn and G Sabidussi, Graph Symmetry: Algebraic Methods and Applications, NATO Advanced Science Institute Series-C 497, Springer, 1997.

[20] R. Hammack, W. Imrich and S. Klavzar, Handbook of Product graphs, CRC Press, 2011.
[21] F. Harary, Graph Theory, Addison-Wesley, 1969.

[22] K. D. Joshi, Applied Discrete Structures, New Age International, New Delhi, 2003.

[23] M B Nathanson, Additive Number Theory, Inverse Problems and Geometry of Sumsets, Springer, New York, 1996.

[24] M B Nathanson, Elementary Methods in Number Theory, Springer-Verlag, New York, 2000.

[25] M B Nathanson, Additive Number Theory: The Classical Bases, Springer-Verlag, New York, 1996.

[26] A. Rosa, On certain valuation of the vertices of a graph, in Theory of Graphs, Gordon and Breach, 1967.

[27] N. K. Sudev and K. A. Germina, On Integer Additie SetIndexers of Graphs, International Journal Mathematical Sciences and Engineering Applications, 8(2)(2014), 11-22.

[28] N. K. Sudev and K. A. Germina, Some New Results on Strong Integer Additive Set-Indexers of Graphs, Discrete Mathematics Algorithms and Applications, 7(1)(2015), 11 pages.

[29] N. K. Sudev and K. A. Germina, (2014). A Characterisation of Strong Integer Additive Set-Indexers of Graphs, Communications in Mathematics and Applications, 5(3), 101-110.

[30] N. K. Sudev and K. A. Germina, A Study on the Nourishing Number of Graphs and Graph Powers, Mathematics, 3(1)(2015), 29-40 .

[31] E. W. Weisstein, CRC Concise Encyclopedia of Mathematics, CRC press, 2011.

[32] D B West, Introduction to Graph Theory, Pearson Education Inc., 2001.

[33] Information System on Graph Classes and their Inclusions, http://www.graphclasses.org/smallgraphs. 